\title{
The Parameters Optimization of MCR-WPT System Based on the Improved Genetic Simulated Annealing Algorithm
}

\author{
Sheng Lu, ${ }^{1}$ Chenyang Zuo, ${ }^{1}$ and Changhao Piao ${ }^{1,2}$ \\ ${ }^{1}$ Institute of Pattern Recognition and Applications, Chong Qing University of Posts and Telecommunications, Chongqing 400065, China \\ ${ }^{2}$ Department of Mechanical Engineering, Inha University, Incheon 402-751, Republic of Korea \\ Correspondence should be addressed to Changhao Piao; changhaopark@hotmail.com
}

Received 13 March 2015; Revised 26 May 2015; Accepted 28 June 2015

Academic Editor: Salvatore Alfonzetti

Copyright (c) 2015 Sheng Lu et al. This is an open access article distributed under the Creative Commons Attribution License, which permits unrestricted use, distribution, and reproduction in any medium, provided the original work is properly cited.

\begin{abstract}
To solve the problem of parameter selection during the design of magnetically coupled resonant wireless power transmission system (MCR-WPT), this paper proposed an improved genetic simulated annealing algorithm. Firstly, the equivalent circuit of the system is analysis in this study and a nonlinear programming mathematical model is built. Secondly, in place of the penalty function method in the genetic algorithm, the selection strategy based on the distance between individuals is adopted to select individual. In this way, it reduces the excess empirical parameters. Meanwhile, it can improve the convergence rate and the searching ability by calculating crossover probability and mutation probability according to the variance of population's fitness. At last, the simulated annealing operator is added to increase local search ability of the method. The simulation shows that the improved method can break the limit of the local optimum solution and get the global optimum solution faster. The optimized system can achieve the practical requirements.
\end{abstract}

\section{Introduction}

Traditional electric power transmission device mainly transmits directly connected via the metal wire, and this kind of connection will cause many problems [1]. With the electric vehicle booming becoming an important green industry, it has received more and more focus [2]. The wireless charging technology could promote the popularity of electric vehicle and solve the problems met in wire charging process. So far, wireless power transmission is divided into electromagnetic induction, magnetic coupling resonant, and radio waves according to the power transmission principle [1]. Electromagnetic induction wireless power through the loosely coupled transformer or discrete transformer is mainly based on the principle of electromagnetic induction [3]. Radio waves wireless power transmission technology is mainly using the principle that the electromagnetic wave power can be transmitted and received through antenna [4]. Magnetic coupling resonant is a new kind of wireless power transmission technology that is proposed by MIT's Professor Marin Soljacic in 2007 [5]. To realize the high effective power transmission, Marin used a couple of transmitting and receiving coils, which have the same resonant frequency, and put the receiving coils in the near field of the transmitting coils. However, radio waves and electromagnetic induction wireless power transmission have the disadvantages of that the transmission power, transmission distance and transmission efficiency cannot reaches to maximum at the same time. By comparison, magnetic coupling resonant wireless power transmission is concerned with the far transmission distance, safety, reliability, high efficiency, and many other advantages. It is considered to be one of the most potential embodiments of wireless power transmission. Nowadays, more and more researchers contribute to this technology $[6,7]$.

The mutual inductance of magnetic coupling resonant wireless power transmission system is very small. In order to improve transmission performance, it requires a high quality factor $\mathrm{Q}$ and $\mathrm{MHz}$ level operating frequency. In addition, it needs to select the most appropriate circuit parameters, because unreasonable parameters selection will damage the device or cause frequency bifurcation [8]. To make the system work stably, reliably, and efficiently, the 


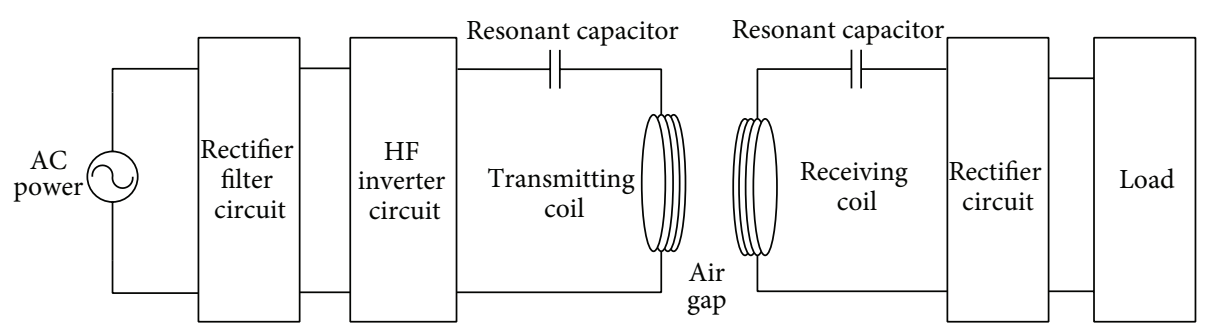

FIGURE 1: The schematic diagram of MCR-WPT system.

parameters must satisfy some constraints. Papers [9-11] have studied the MCR-WPT parameters' design. These studies have not taken the constraints of the parameters into account comprehensively. Usually, low efficiency is generated by using stepwise design method. Magnetic coupling resonant wireless power transmission system is a kind of multiparameter and multiconstrained nonlinear system [12]. The complexity of the system equation will increase with the number of the coils. When the traditional optimization methods are used to solve the system, the system model needs to be simplified. The traditional methods are based on the system function model derivative, which does not fully consider the constraints of the components' working conditions for the optimized parameters, so the parameters cannot be directly used in practice and need to be further amended. Nevertheless, only one parameter is optimized in every amendment, not all of them. As a result, it cannot guarantee getting global optimal solution when making a few parameters together in this way.

In order to achieve the maximums of transmission efficiency and distance, respectively, this paper fully considers the constraints of the system to establish the mathematical model and proposes an improved genetic simulated annealing algorithm to optimize parameters simultaneously. Genetic algorithm (GA), which does not require that the objective function is derivative, can be optimized in multiparameter and multiconstraint conditions. Therefore, it shows a good performance on robustness [13]. GA performs better in global search than in local search. Although GA is able to search the globally optimal solution in a random way based on probability, in reality, there exist problems such as premature phenomenon and low ability in local optimization. To improve the searching abilities of GA, firstly, the selection strategy based on the distance between individuals was used. Thus, it can avoid the loss of population diversity and the fitness value can be calculated according to feasibility-based rules. Also, it simplifies the setting of too many parameters. Then, the method of calculating crossover probability $\left(P_{c}\right)$ and mutation probability $\left(P_{m}\right)$ according to the variance of population fitness makes $P_{c}$ and $P_{m}$ change with the increasing of iteration. It really makes sense of raising the rate of convergence. Simulated annealing algorithm (SA) simulates the process of metal annealing, which can reach a commendable result by setting a high initial temperature and a slow process of annealing. SA has a good ability of local search as well as convergence of globally optimal solution. Meanwhile, SA is able to break away from the local optimal solution and restrain the premature phenomenon. Improved genetic simulated annealing algorithm (IGSA) combines the advantages of GA and SA. At the same time, it adds an improved selection strategy and $P_{c}$ and $P_{m}$ strategy which can increase the ability of searching and the rate of convergence. IGSA is used for parameters optimization design for the series-series system structure. The comparison shows that the optimization ability of IGSA is better than the general algorithm and ANSYS finite-element software is used for simulation verification.

\section{The Mathematical Model of the MCR-WPT System}

As shown in Figure 1, the transmitter of magnetically coupled resonant wireless power transfer system is composed of AC power, rectifier filter circuit, high-frequency inverter circuit, transmitting coil, and resonant capacitor. Rectifier filter circuit converts alternating current (AC) to direct current (DC) for high-frequency inverter circuit, and then it will output high-frequency current to transmitting coil. The receiver consists of receiving coil, resonant capacitor, rectifier circuit, and load. Rectifier filter circuit converts alternating current $(\mathrm{AC})$ occurring in receiving coil to direct current (DC) for load. On special occasion, DC-DC converter may be introduced into the rectifier filter circuit to convert the high voltage and small current input into large current and low voltage output $[14,15]$. The power between transmitting terminal and receiving end transfers through the magnetic coupling resonance.

The analysis object of this paper is the transmitter seriesthe receiver series topology. The equivalent circuit is shown in Figure 2. $L_{p}$ and $L_{s}$, respectively, represent transmitting coil and receiving coil's equivalent inductances. $R_{1}$ and $R_{2}$, respectively, represent the equivalent resistances of transmitting coil and receiving coil. $U_{s}$ is the output electromotive force of source of the inverter circuit and $R_{s}$ is the output resistance of the inverter circuit. $C_{p}$ and $C_{s}$ indicate the resonant capacitor of transmitter and the receiver. $I_{p}$ and $I_{s}$ are the current of the lunching circuit and receiving circuit. $R_{L}$ is load resistance. $M$ is the mutual inductance between transmitting coil and receiving coil. $D$ expresses the distance of transmitting coil and receiving coil. When we add $U_{s}$ to the ends of the transmitting coil, it can produce the same frequency in the coil magnetic field. Receiving coil is in the near field region of transmitting coil and these two coils have the same frequency. As a result, it can create magnetic coupling resonance. 


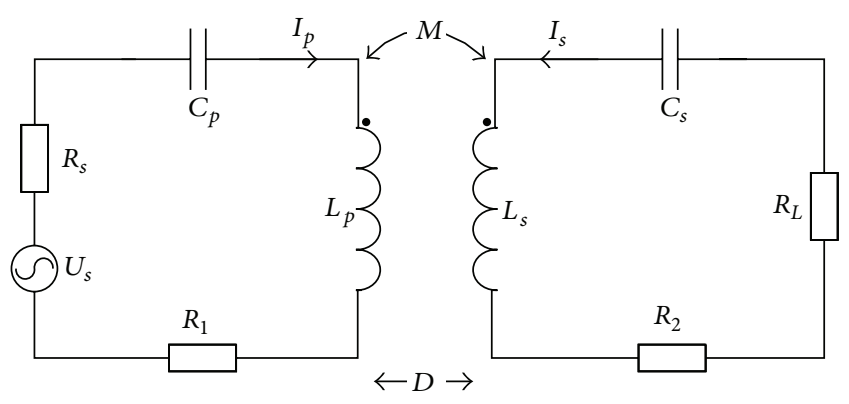

FIgURE 2: The equivalent circuit of SS structure.

According to the model of mutual inductance equivalent circuit, we can establish the following equations:

$$
\begin{gathered}
U_{s}=j \omega M I_{s}+j\left(\omega L_{p}-\frac{1}{\omega C_{p}}\right) I_{p}+\left(R_{s}+R_{1}\right) I_{p}, \\
0=j \omega M I_{p}+j\left(\omega L_{s}-\frac{1}{\omega C_{s}}\right) I_{s}+\left(R_{L}+R_{2}\right) I_{s} .
\end{gathered}
$$

In the actual system, for ease of design and analysis, the transmitting coil and receiving coil are all designed as the parallel and coaxial structure. Meanwhile, the influence of coil resistances $R_{1}$ and $R_{2}$ is ignored. The self-inductance of the transmitting coil and receiving coil is $L$, and impedance of the transmitting loop and receiving loop is, respectively, represented by $Z_{p}$ and $Z_{s}$. The impedance reflecting from the receiving end to the transmitting end is $Z_{r}$. And the mutual inductance of the transmitting coil and receiving coil is $M$ [5]. Thus, there exists the following relationship:

$$
\begin{aligned}
L & =L_{p}=L_{s} \approx N^{2} \mu_{0} r\left[\ln \left(\frac{8 r}{a}\right)-2\right], \\
M & \approx \frac{\pi \mu_{0} N r^{4}}{2 D^{3}} \\
\omega_{0} & =\frac{1}{\sqrt{L_{p} C_{p}}}=\frac{1}{\sqrt{L_{s} C_{s}}}=\frac{1}{\sqrt{L C}} \\
Z_{p} & =R_{s} \\
Z_{s} & =R_{L} \\
Z_{r} & =\frac{\omega_{0}^{2} M^{2}}{Z_{s}}
\end{aligned}
$$

where $N, a, r$, and $D$, respectively, represent the loop number, coil wire diameter, coil radius, and the distance of coils. The current of the receiver circuit is

$$
I_{s}=\frac{-j \omega_{0} M I_{p}}{Z_{s}} .
$$

The current of the transmitter circuit is

$$
I_{p}=\frac{U_{s}}{Z_{r}+Z_{p}}=\frac{U_{s} Z_{s}}{\omega_{0}^{2} M^{2}+Z_{p} Z_{s}} .
$$

The power of the load is

$$
P_{L}=I_{s}^{2} R_{L}=\left(\frac{\omega_{0} M I_{p}}{Z_{s}}\right)^{2} R_{L}
$$

The power of the main input is

$$
P_{\text {in }}=U_{s} I_{p}=\frac{U_{s}^{2} R_{L}}{\omega_{0}^{2} M^{2}} .
$$

The efficiency of the transmission system is

$$
\eta=\frac{P_{L}}{P_{\text {in }}}=\frac{\left(\omega_{0} M\right)^{2} R_{L}}{Z_{s}\left[\left(\omega_{0} M\right)^{2}+Z_{p} Z_{s}\right]} \times 100 \% .
$$

According to the frequency and quality factor of system, paper [16] has studied the influence of transmission power. The critical coupling coefficient is proposed as $k_{c}=$ $\sqrt{\left(Q_{p}^{2}+Q_{s}^{2}\right) / 2 Q_{p}^{2} Q_{s}^{2}}$. When the coupling coefficient $k<$ $k_{c}$, transmission power reaches to maximum at resonant frequency point $\omega_{0}$. But with the decrease of $k$, the maximum power will be with much attenuation. If the coupling coefficient $k<k_{c}$, the maximum transmitted power will appear on both sides of $\omega_{0}$. By this time, with the decrease of $k$, the maximum transmission power point will be closer to $\omega_{0}$. A little deviation from $\omega_{0}$ will not make transmission power decline. In order to control the working frequency of the system easily at a resonance point and ensure the transmission distance and high transmission efficiency, the coupling coefficient $k$ of SS system needs to satisfy the conditions as follows:

$$
0.8 \sqrt{\frac{Q_{p}^{2}+Q_{s}^{2}}{2 Q_{p}^{2} Q_{s}^{2}}}<\frac{M}{\sqrt{L_{p} L_{s}}}<1.1 \sqrt{\frac{Q_{p}^{2}+Q_{s}^{2}}{2 Q_{p}^{2} Q_{s}^{2}}},
$$

where $Q_{p}=\omega_{0} L_{p} / R_{s}$ is the quality factor of transmitter circuit and $Q_{s}=\omega_{0} L_{s} / R_{L}$ is the quality factor of receiver circuit. So, as is shown in (8), if the excitation frequency is controlled as $\omega_{0}$, high transmission power and distance will be obtained. As mentioned above, a little deviation from $\omega_{0}$ will not influence the transmission power apparently.

Let $V_{C_{p} \text {-rate }}$ and $V_{C_{s} \text {-rate }}$ represent the rated voltage of $C_{p}$ and $C_{s}$. The rated value of $I_{p}$ and $I_{s}$, in other words the minimum rated current of $C_{p}$ and $C_{s}$, respectively, is represented by $I_{p \text {-rate }}$ and $I_{s \text {-rate }}$. The parameters of the system need to satisfy the following constraints:

$$
\begin{aligned}
V_{C_{p}} & =\frac{I_{p}}{\left(\omega_{0} C_{p}\right)}<V_{C_{p} \text {-rate }} \\
V_{C_{s}} & =\frac{I_{s}}{\left(\omega_{0} C_{s}\right)}<V_{C_{s} \text {-rate }} \\
I_{p} & <I_{p \text {-rate }}, \\
I_{s} & <I_{s \text {-rate }},
\end{aligned}
$$


where $V_{C_{p}}$ and $V_{C_{s}}$, respectively, represent the voltage of $C_{p}$ and $C_{s}$. According to the actual working situation, $C_{p}$ and $C_{s}$ have the maximum values $C_{p \text {-max }}$ and $C_{s-\max }$ and the minimum values $C_{p-\min }$ and $C_{s-\min }$. There are size restrictions in coil; that is to say, the turns and radius of coil have the maximum values $N_{\max }$ and $r_{\max }$ and the minimum values $N_{\min }$ and $r_{\min }$. The switch tube of high-frequency inverter circuit has the biggest switching frequency which is the maximum frequency $f_{0 \text {-max }}$ of the AC power excitation. Meanwhile, the minimum working frequency $f_{0 \text {-min }}$ is set. As maximum distance value $D_{\max }$ and minimum distance value $D_{\min }$ exist between transmission coil and receiving coil according to the working condition of lord and transmitting terminal, the constricts of each parameter can be expressed in

$$
\begin{gathered}
C_{p-\min }<C_{p}<C_{p-\max }, \\
C_{s-\min }<C_{s}<C_{s-\max }, \\
N_{\min }<N<N_{\max }, \\
r_{\min }<r<r_{\max }, \\
f_{0 \text {-min }}<f_{0}<f_{0 \text {-max }}, \\
D_{\text {min }}<D<D_{\max } .
\end{gathered}
$$

The maximum, minimum, and rated values of $R_{L}, R_{s}$, $U_{s}$, and the above parameters are known. In order to reach the highest transmission efficiency and transmission distance of the system, the parameters $N, r, D$, and $\omega_{0}=2 \pi f_{0}$ are optimized. The optimized objective function is as follow:

$$
\begin{aligned}
& \min f_{\mathrm{obj}}=-\left(\frac{D-D_{\min }}{D_{\max }-D_{\min }}+\eta\right) \\
& =-\left(\frac{D-D_{\min }}{D_{\max }-D_{\min }}+\frac{\omega_{0}^{2} \pi^{2} \mu_{0}^{2} N^{2} r^{8}}{\omega_{0}^{2} \pi^{2} \mu_{0}^{2} N^{2} r^{8}+4 D^{6} R_{s} R_{L}}\right) .
\end{aligned}
$$

Inserting (2)-(4) into (8)-(10), we get the constriction of the system:

$$
\begin{aligned}
g_{1}= & \sqrt{2} \pi \mu_{0} N r^{4} \omega_{0}-2.2 D^{3} \sqrt{R_{s}^{2}+R_{L}^{2}}<0, \\
g_{2}= & 1.6 D^{3} \sqrt{R_{s}^{2}+R_{L}^{2}}-\sqrt{2} \pi \mu_{0} N r^{4} \omega_{0}<0, \\
g_{3}= & 4 U_{s} R_{L} \mu_{0} \omega_{0} r D^{6} N^{2}\left[\ln \left(\frac{8 r}{a}\right)-2\right] \\
& -V_{C_{p} \text {-rate }}\left(\omega_{0}^{2} \pi^{2} \mu_{0}^{2} N^{2} r^{8}+4 D^{6} R_{s} R_{L}\right)<0, \\
g_{4}= & 2 U_{s} \pi \mu_{0}^{2} \omega_{0}^{2} D^{3} N^{3} r^{5}\left[\ln \left(\frac{8 r}{a}\right)-2\right] \\
& -V_{C_{s} \text {-rate }}\left(\omega_{0}^{2} \pi^{2} \mu_{0}^{2} N^{2} r^{8}+4 D^{6} R_{s} R_{L}\right)<0, \\
g_{5}= & 4 D^{6} U_{s} R_{L}-I_{C_{p} \text {-rate }}\left(\omega_{0}^{2} \pi^{2} \mu_{0}^{2} N^{2} r^{8}+4 D^{6} R_{s} R_{L}\right) \\
< & 0, \\
g_{6}= & 2 U_{s} \pi \mu_{0} \omega_{0} N D^{3} r^{4} \\
& -I_{C_{s} \text {-rate }}\left(\omega_{0}^{2} \pi^{2} \mu_{0}^{2} N^{2} r^{8}+4 D^{6} R_{s} R_{L}\right)<0,
\end{aligned}
$$

$$
\begin{aligned}
& g_{7}=1-C_{\max } \omega_{0}^{2} N^{2} \mu_{0} r\left[\ln \left(\frac{8 r}{a}\right)-2\right]<0, \\
& g_{8}=C_{\min } \omega_{0}^{2} N^{2} \mu_{0} r\left[\ln \left(\frac{8 r}{a}\right)-2\right]-1<0 .
\end{aligned}
$$

\section{MCR-WPT System Parameters Optimization Based on Improved Genetic
Simulated Algorithm}

Genetic algorithm achieves a good performance in global search, but it is easy to trap into local optimal solution. Simulated annealing algorithm performs well in local search, but its convergence speed is slow. To solve the parameters optimization problems of systems with multiple constraints and parameters, the advantages of the two algorithms are combined. The idea of using genetic simulated algorithm to optimize parameters of MCR-WPT system is described as follows. Firstly, the feasibility-based rules are applied to selecting operation to prevent the loss of population diversity. Secondly, to improve the search capability and convergence speed of the algorithm, population fitness variance is used to calculate crossover probability and mutation probability. The heuristic cross and nonuniform mutations are used for individual crossover and mutation operation. Finally, the simulated annealing operation is carried out to prevent the algorithm from early convergence.

3.1. Improved Selection Strategy. Deb proposed a method to handle GA feasibility selection operation [17]. The key point is that the method can solve feasible solutions. The main requirements of the method are as follows: (1) the feasible solution is superior to the nonfeasible solution; (2) comparing the two feasible solutions, the one with optimal objective value is desired; (3) comparing the two nonfeasible solutions, the one with low degree of default function is desired. Compared with penalty function method, there are no extra empirical parameters used in the method, and a championship method is used to select the superior individuals. But the method falls into local solution easily. Because of the neglect of nonfeasible solution, the evolutional population is mainly composed of feasible solutions. Thus, the similarity of population individuals is quite high and the individuals are relatively single. Through every selecting operation, the individuals of next generation are mainly feasible ones, which reduce the population diversity. To keep population diversity, the selected individuals should avoid being all feasible ones or feasible and nonfeasible ones. In this paper, the selection strategy is improved. The selecting operation of feasibility for individuals is decided by the distance-based method.

Firstly, to calculate Euclidean distance and complete normalization processing, two individuals $i$ and $j$ are chosen randomly to form the population. It can be shown as

$$
d_{i, j}=\sqrt{\frac{\sum_{k=1}^{n}\left(x_{k}^{i}-x_{k}^{j}\right)^{2}}{\sum_{k=1}^{n}\left(x_{k}^{\max }-x_{k}^{\min }\right)^{2}}},
$$


where $x_{k}^{i}$ and $x_{k}^{j}$ are, respectively, the $k_{\text {th }}$ component values of individuals $i$ and $j$ and $x_{k}^{\max }$ and $x_{k}^{\min }$ represent the maximum and minimum of the $k_{\mathrm{th}}$ component, respectively. By judging whether the $d_{i, j}$ is smaller than $h, h \in(0,1)$, the larger the value $h$ is, the smaller the similarity of the individuals $i$ and $j$ is. And the smaller the value $h$ is, the larger the similarity of the individuals $i$ and $j$ is. If the condition does not satisfy the judgment, the individual is to be selected again. If it does, the individual fitness will be calculated according to

$$
\begin{aligned}
& F(X) \\
& \quad= \begin{cases}f(x) & \text { if } g_{i}(X)<0, i=1, \ldots, m \\
f_{\max }+\sum_{j=1}^{m}\left|g_{i}(X)\right| & \text { otherwise, }\end{cases}
\end{aligned}
$$

where $f_{\max }$ is the objective function of the worst individual among the current feasible solutions and $m$ is the number of constraint conditions. Lastly, according to Deb's feasible rule, these two individuals are compared and we can determine which individual will enter the crossover and mutation operations of next generation. Because similar individuals are compared every time, population diversity is kept. Thus, it will be conducive to individuals distributing in a wide range space and contribute to global search.

3.2. Improved Crossover and Mutation Operation. At the beginning, the algorithm needs strong global search ability and weak local search ability, which are helpful for searching in global range. Thus, at the early stage of the algorithm, the crossover probability $P_{c}$ is relatively high and mutation probability $P_{m}$ is relatively small. At the late stage of the algorithm, in order to converge to optimization solution, the global search ability needs to reduce and local search to be strengthened. So, at the late stage of the algorithm, the crossover probability $P_{c}$ is relatively small and mutation probability $P_{m}$ is relatively high. It can avoid precocious phenomenon. Combining with logistic mathematical equation, the methods of calculating $P_{c}$ and $P_{m}$ can be obtained by

$$
\begin{aligned}
P_{c} & =\frac{1}{1+e^{1 / x}}+0.4, \\
P_{m} & =\frac{0.3}{1+e^{-1 / x}},
\end{aligned}
$$

where $x$ is the variance of population fitness and crossover probability $P_{c}$, whose available interval is $[0.4,0.88]$, decreases according to the decrease of $x$. On the contrary, the mutation probability $P_{m}$, whose available interval is $[0.16,0.3]$, increases according to the decrease of $x$. With the deep evolution, the individuals are more integrated, which shows that $x$ is smaller and smaller and the variation tendency of $P_{c}$ and $P_{m}$ conforms to the rules of genetic algorithm.
The crossover operation uses heuristic crossover and selects $x_{i}$ and $x_{j}$ as the individuals needing crossover operation according to crossover probability $P_{c}$ [18]. The new individuals after crossover operation are $x_{i}^{\prime}$ and $x_{j}^{\prime}$; that is,

$$
\begin{aligned}
& x_{i}^{\prime}=x_{j} \quad \text { if individual } x_{j} \text { is better than } x_{i}, \\
& x_{j}^{\prime}=x_{j}+\alpha *\left(x_{i}-x_{j}\right) \quad \text { otherwise, }
\end{aligned}
$$

where $\alpha=G_{i} / G_{\max }, G_{i}$ is the current algebra, and $G_{\max }$ is the maximum algebra.

The crossover operation changes with the deep evolution, and the crossover effect gradually decreases. This crossover operation is conducive to global search in the early evolution and local optimization solution search in the late mutation operation. Meanwhile, it can prevent the excellent individuals from being damaged.

Mutation operation determines the local search ability of algorithm. In this paper, we use nonuniformity mutation operator, which is proposed by Michalewicz for floatingpoint encoding, to do the operation of individuals mutating [19]. The new individuals after mutation operation are $x_{i}^{\prime}$ :

$$
x_{i}^{\prime}= \begin{cases}x_{i}+\left(b_{i}-x_{i}\right)\left(r_{1}\left(1-\frac{G_{i}}{G_{\max }}\right)\right) & r_{2}<0.5 \\ x_{i}-\left(a_{i}-x_{i}\right)\left(r_{1}\left(1-\frac{G_{i}}{G_{\max }}\right)\right) & r_{2} \geq 0.5,\end{cases}
$$

where $x_{i} \in\left[a_{i}, b_{i}\right], a_{i}$ is the lower bound of $x$, and $b_{i}$ is the upper bound of $x, r_{1}$ and $r_{2}$ is random number between 0 and 1 .

The mutation step in the operator decreases adaptively with the deep evolution. In the early stage of iteration, the big mutation step improves search efficiency. In the late stage, the small mutation step search keeps the optimal solution.

3.3. Simulated Annealing Operation. The individuals after crossover and mutation operation use simulated annealing operation. The Metropolis criterion is used to accept those new individuals. The specific operations are as follows. The new individual $x^{\prime \prime}$ is generated from the domain $N\left(x^{\prime}\right)$ of individual $x^{\prime}$, and $\Delta f=f\left(x^{\prime}\right)-f\left(x^{\prime \prime}\right)$. is calculated. If $\Delta f \leq 0$, the new individual $x^{\prime \prime}$ replaces the old individual $x^{\prime}$; namely, $x^{\prime}:=x^{\prime \prime}$. If $\Delta f>0, e^{-\Delta f / t_{k}}>\operatorname{random}(0,1)$ will be calculated. If $e^{-\Delta f / t_{k}}>\operatorname{random}(0,1)$ is true, the new individual will be accepted.

The cooling operation in the simulated annealing algorithm is as follows:

$$
T(k+1)=r * T(k),
$$

where $r$ is positive constant, slightly less than 1 , and $k$ is cooling times. $T(0)$ indicates the initial temperature and usually is a very large value.

The simulated annealing operation does not always negate nonfeasible solution but would accept it in a certain probability. This is conducive to solve the problem of search stagnation in the late stage of the algorithm and prevent the optimization results from locally optimal solution. 


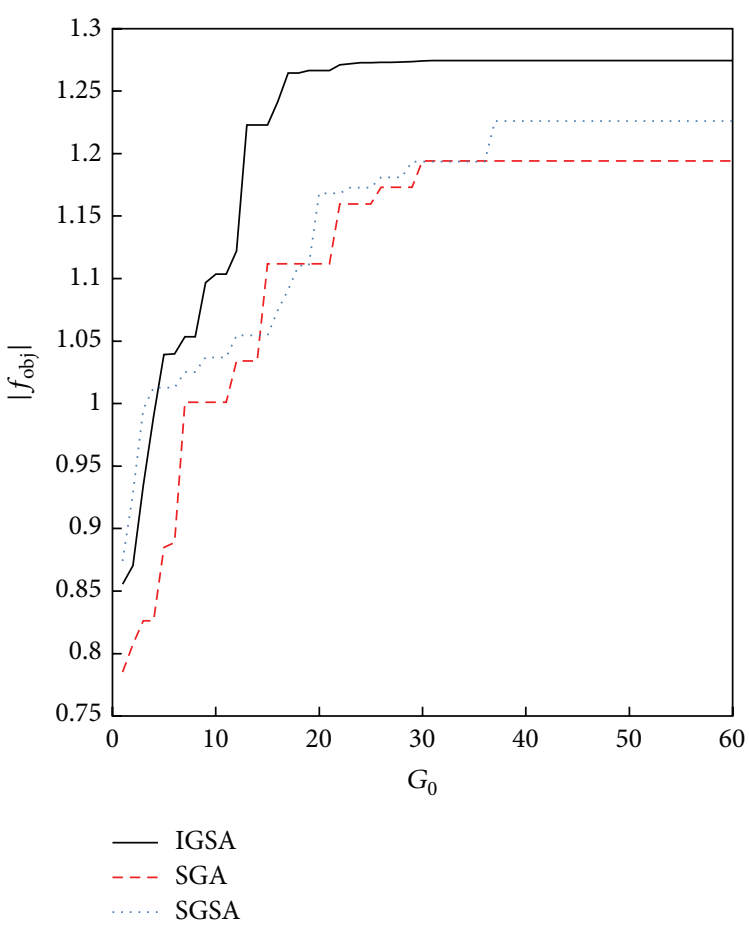

(a) The initial population of 100

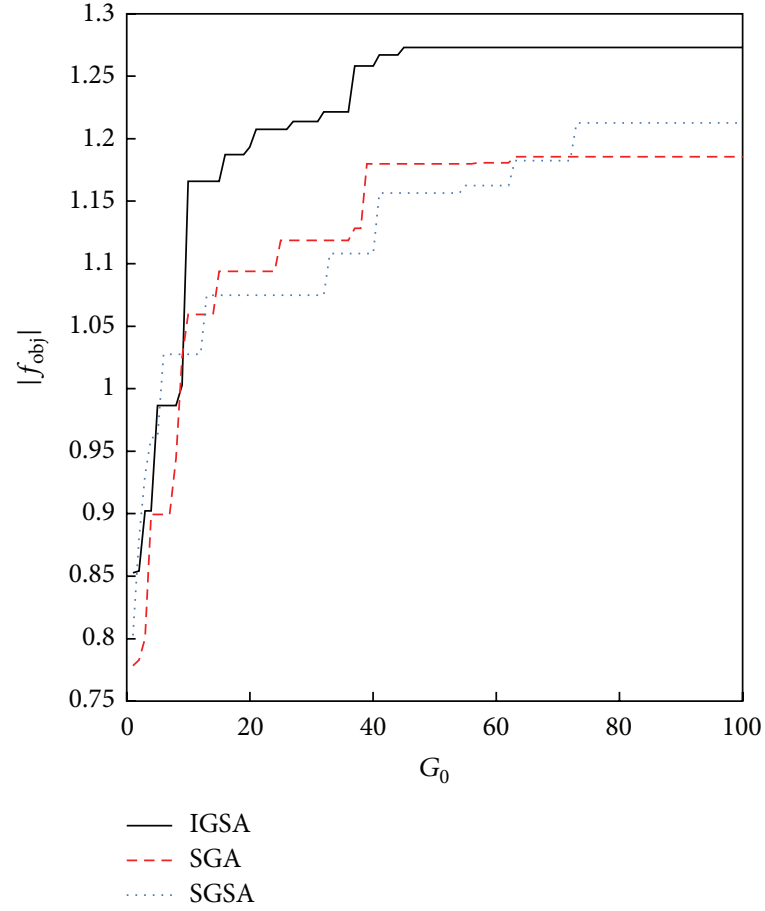

(b) The initial population of 150

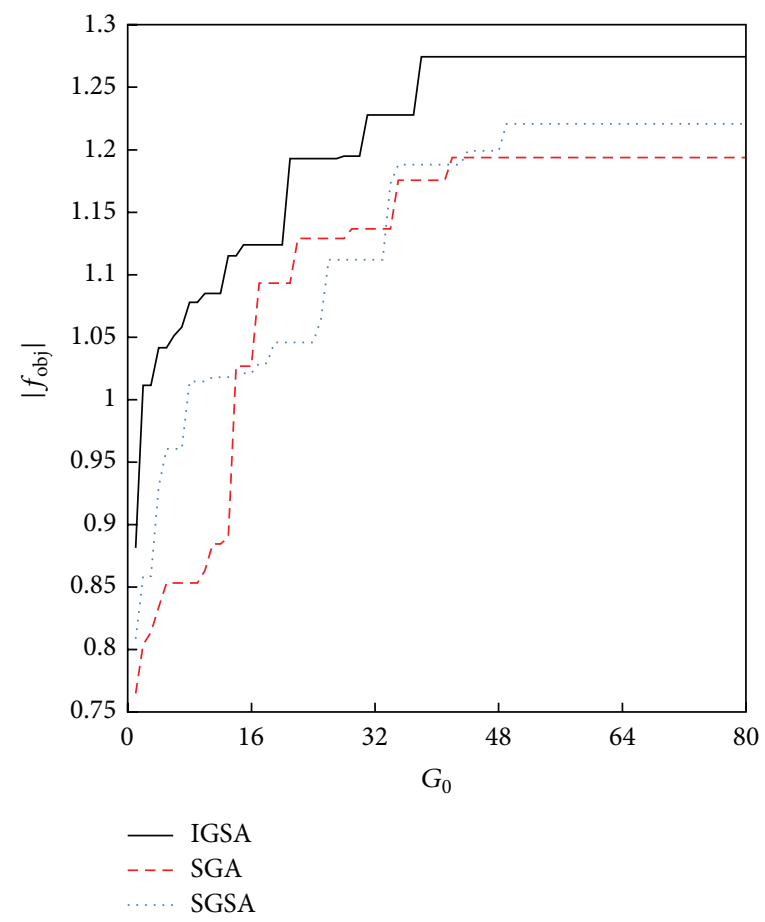

(c) The initial population of 200

FIGURE 3: The solved objective function curves with different initial population.

\section{MCR-WPT System of Simulations and Finite-Element Verification}

In this section, three methods including simple genetic algorithm (SGA), simple genetic simulated annealing algorithm (SGSA), and IGSA have been applied to optimize system's parameters. With the initial population of 100, 150, and 200, respectively, the optimal optimized results are shown in Figure 3. The specifications of the magnetic coupling resonant wireless power transmission system based on SS topology are provided in Table 1. 

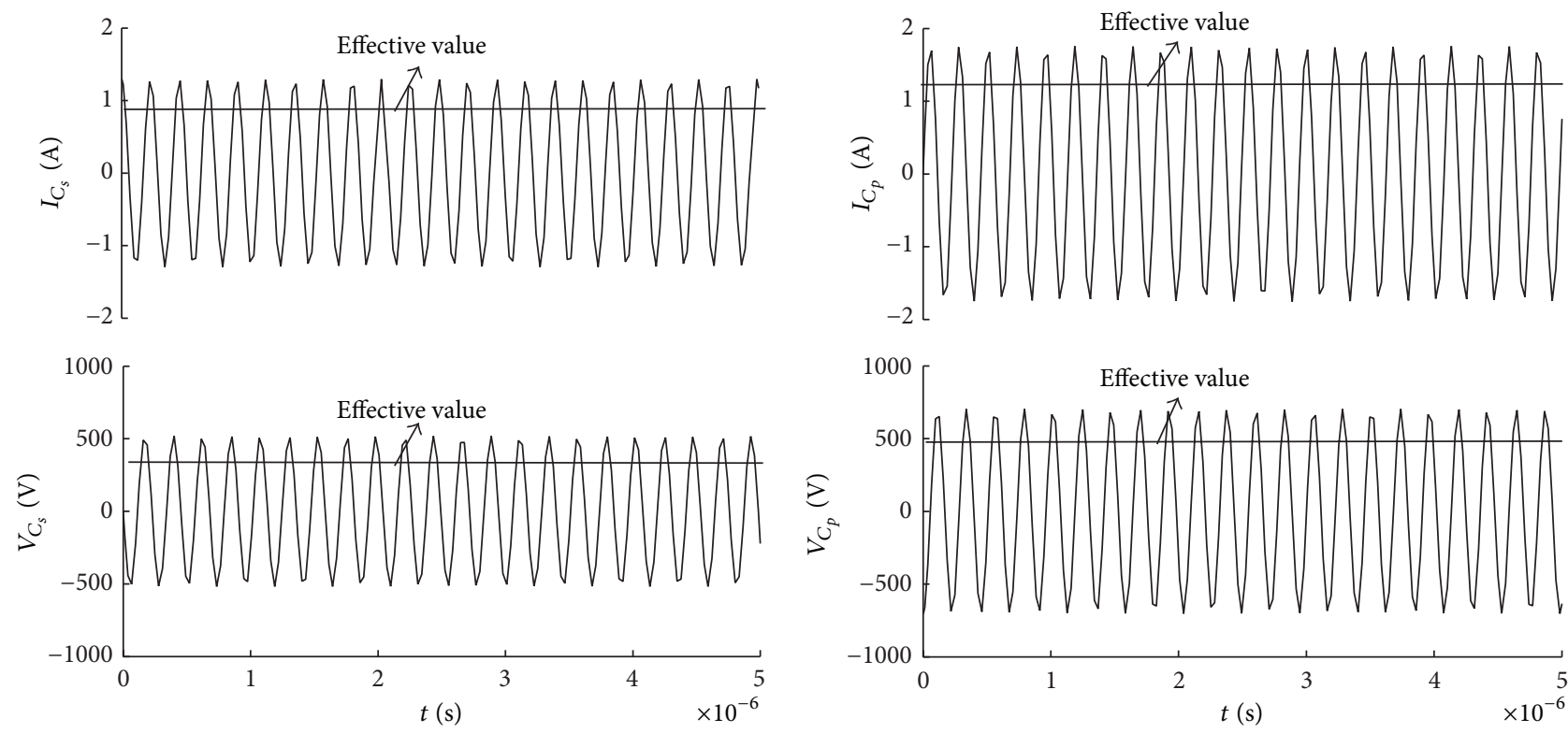

FIGURE 4: The voltage and current waveforms of the transmitting and receiving coils with optimal parameters.

TABLE 1: The parameter values of WCR-WPT system.

\begin{tabular}{lc}
\hline Parameters & Nominal value \\
\hline$R_{L} / \Omega$ & 50 \\
$R_{s} / \Omega$ & 5 \\
$U_{s} / \mathrm{V}$ & 40 \\
$a / \mathrm{mm}$ & 1.6 \\
$V_{C_{s-\text { rate }}}$ and $V_{C_{p \text {-rate }} / \mathrm{V}} / \mathrm{A}$ & 500 \\
$I_{p \text {-rate }} / \mathrm{A}$ & 4 \\
$I_{s-\text { rate }}$ & 3 \\
$C_{s}$ and $C_{p} / \mathrm{nF}$ & {$[0.09,3]$} \\
$N$ & {$[2,10]$} \\
$r / \mathrm{mm}$ & {$[50,80]$} \\
$D / \mathrm{mm}$ & {$[50,100]$} \\
$f / \mathrm{KHz}$ & {$[2000,5000]$} \\
\hline
\end{tabular}

From Figure 3, it is known that the rate of convergence of the system with SGA is small. It plunges into local optimum easily and the optimization is useless. Also, we can see that the local search ability of the system is enhanced by SGSA. However, the rate of convergence is small and the optimization effect is worse than using IGSA. Thanks to improved selection strategy of individuals and calculation method of $P_{C}$ and $P_{m}$, not only the local search ability of the system improves observably, but also the rate of convergence changes faster and the optimization is better than using the other two methods.

With the initial population of 200 , the optimized maximum is $f_{\text {obj }}=1.2744$ and the transmission efficiency is $\eta=84.4 \%$. And the corresponding optimal parameters are $r=80 \mathrm{~mm}, \omega_{0}=27.73 \times 10^{6} \mathrm{rad} / \mathrm{s}\left(f_{0}=4414.2 \mathrm{kHz}\right)$, $N=6$, and $D=71.5 \mathrm{~mm}$. By substituting these parameters into (10), it is known that they meet the constraint conditions.
According to optimal parameters, the other parameters are obtained; that is, $L_{p}=L_{s}=L=14.5 \mu \mathrm{H}, C_{p}=C_{s}=C=$ $89.7 \mathrm{pF}$, and $M=1.33 \mu \mathrm{H}$. Further, based on these values, the model and simulations are achieved by Simulink. Figure 4 shows the voltage and current waveforms of the transmitting and receiving coils. It is clear that $I_{p}, I_{s}, V_{C_{p}}$, and $V_{C_{s}}$ are 1.24 A, 0.92 A, 497.4 V, and $366.5 \mathrm{~V}$, respectively. Obviously, the capacitor voltage and current satisfy the requirements.

The finite-element method is usually applied in force and electromagnetism analysis [20]. In order to verify the consistency between coil inductance and mutual inductance when the coil has a feature with $N=6, r=80 \mathrm{~mm}$, and $a=1.6 \mathrm{~mm}$, we use ANSYS to simulate the system. In this paper, PLANE53 element is selected as the element attribute of coil and air, and an axisymmetric model was employed for system modeling. The finite-element mesh generation for the established model is shown in Figure 5. After exerting boundary conditions and excitation, we get $L=12.9 \mu \mathrm{H}$ and $M=1.3 \mu \mathrm{H}$ by LMATIRX. Obviously, the differences between the simulated values and the theoretical values are very small. The reason for small differences is to simplify the coil model. The cross section of the coil model is rectangle, which is larger than the actual coil.

Figure 6 shows the system's finite-element model with PLANE53 and CIRCUIT124 elements. Based on the optimal parameters and adopting ANSYS electromagnetic analysis, the simulation result is shown in Figure 7. As the figure shows, the coupling degree of the resonance point is weaker than that of the unresonant points. But the overall intensity of the magnetic field in the resonance point is more than 2 times larger than that in the unresonant point.

Through ANSYS postprocessing function, the values of input power and load power in each frequency point are extracted. So the curve of the relationship between the transmission efficiency and frequency can be obtained. As 


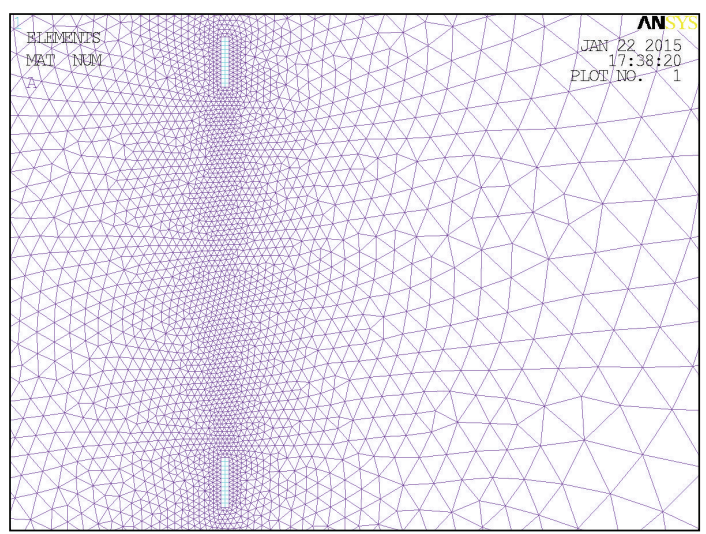

FIGURE 5: The coil model with finite-element mesh generation.

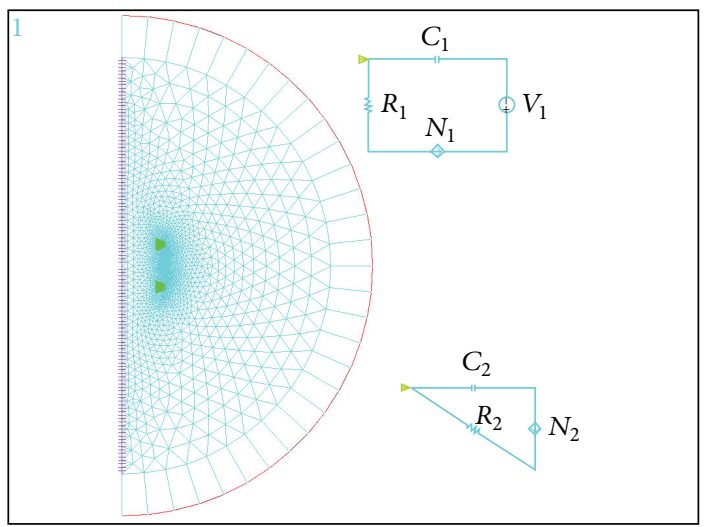

FIGURE 6: The finite-element model of power transfer system.

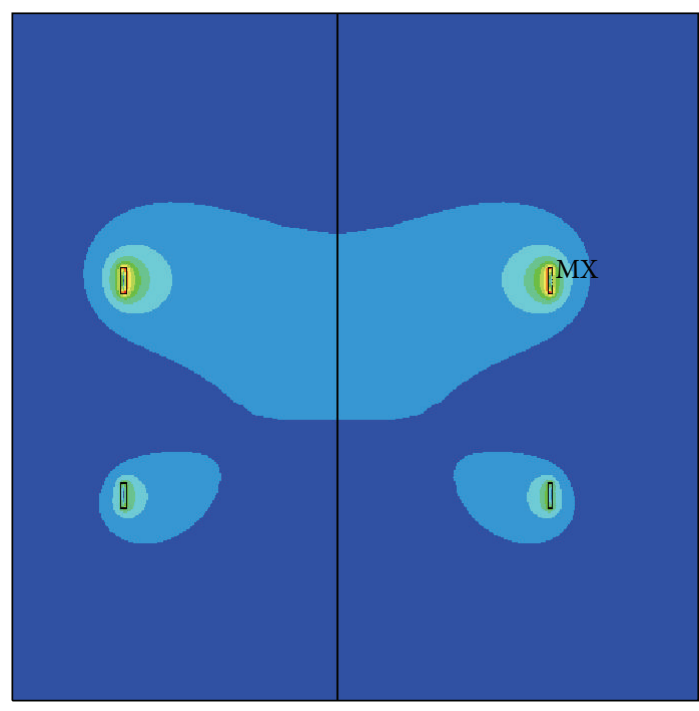

$T$ (tesla)

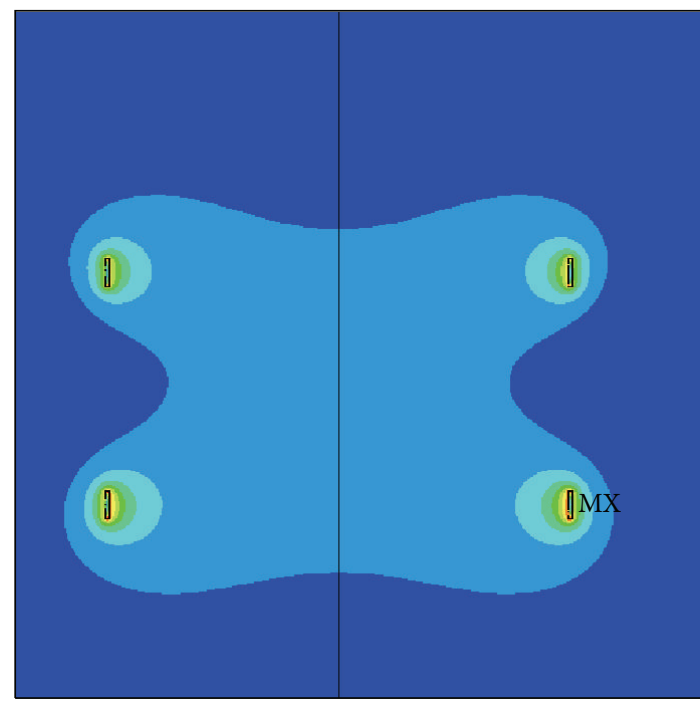

$T$ (tesla)

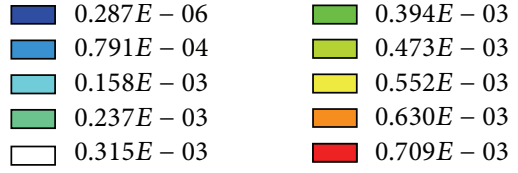

(a)

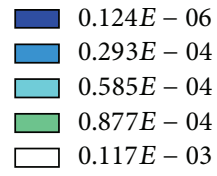

$0.117 E-03$

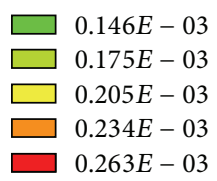

(b)

FIgURE 7: The magnetic field distributions of resonant point (a) and unresonant point (b), respectively. 


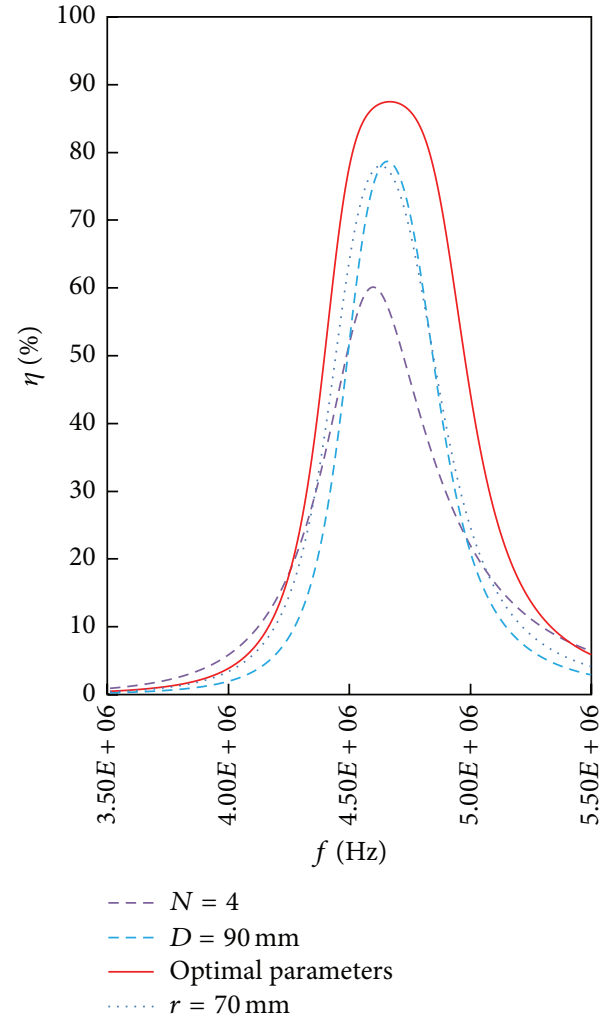

FIgURE 8: The relationships of $\eta$ with $f$ under the different parameters.

is shown in Figure 8, the transmission efficiency reached the maximum at the resonant frequency. Due to the small simulated inductance value, the resonant frequency would be larger than the theoretical value.

In order to validate that the transmission efficiency can reach the maximum based on the optimal parameters, without considering the constraint conditions, one of the parameters is changed while the others are kept constant. In this way, three transmission efficiency curves can be obtained as shown in Figure 8. Obviously, system transmission efficiency reaches the maximum by using the optimal parameters. In the meantime, the efficiency curve crest is relatively flat. So the attenuation changes inconspicuously with the deviation of the resonance point at $\pm 100 \mathrm{Khz}$. But, for the other three parameters, the transmissions efficiencies never reached the maximum and the efficiency curve crests are all very sharp. So the attenuation is influenced apparently if a little deviation of resonant frequency point appears. According to the analysis above, the system can achieve a good transmission performance by using the optimal parameters. Meanwhile, the system can control the frequency easily. It fully meets the system design requirements.

\section{Conclusion}

In this paper, at first, by analyzing the SS topology of the MCR-WPT system, the parameters expressions are obtained and the corresponding boundary conditions and objective functions are established. Then, IGSA is used to optimize the system's parameters. The proposed algorithm can decide which individuals would be chosen for the next genetic manipulation by calculating the distances among individuals. The method increases the population individual multiplicity and prevents local convergence. Besides, it can adjust the crossover and mutation probabilities based on the fitness variances of the individuals in every generation. Thus, the convergence speed increases. Finally, simulated annealing operation for progeny is implemented to improve the search ability of the algorithm. The ANSYS finite-element model is designed to verify the optimized results. It verifies that the system with optimal parameters can achieve optimal performances and it can control the frequency easily.

\section{Conflict of Interests}

The authors declare that there is no conflict of interests regarding the publication of this paper.

\section{Acknowledgments}

This work is supported by the Foundation of Chongqing Science and Technology Commission (CSTC 2013jcyjjq60002, CSTC 2013yykfC60005, and CSTC 2013 jcsf-jcssX0022) and the National Natural Science Foundation of China (NSFC 11247325).

\section{References}

[1] Z. M. Zhao, Y. M. Zhang, and K. N. Chen, "New progress of magnetically-coupled resonant wireless power transfer technology," Proceedings of the Chinese Society of Electrical Engineering, vol. 33, no. 3, pp. 1-13, 2013.

[2] Y. Xie, Z. G. Wang, and Y. S. Zhang, "Development of battery system testing machine," International Journal of Future Engineering, vol. 2015, Article ID 3315101, 7 pages, 2015.

[3] Y.-S. Seo, M. Q. Nguyen, Z. Hughes, S. Rao, and J.-C. Chiao, "Wireless power transfer by inductive coupling for implantable batteryless stimulators," in Proceedings of the IEEE MTT-S International Microwave Symposium Digest, pp. 1-3, Montreal, Canada, June 2012.

[4] M. Ali, G. Yang, and R. Dougal, "A miniature packaged rectenna for wireless power transmission and data telemetry," in Proceedings of the IEEE International Workshop on Antenna Technology Small Antennas and Novel Metamaterials (IWAT '06), pp. 225-228, IEEE, March 2006.

[5] A. Kurs, A. Karalis, R. Moffatt, J. D. Joannopoulos, P. Fisher, and M. Soljačić, "Wireless power transfer via strongly coupled magnetic resonances," Science, vol. 317, no. 5834, pp. 83-86, 2007.

[6] S.-H. Lee and R. D. Lorenz, "Development and validation of model for 95\%-efficiency 220 -W wireless power transfer over a 30-cm air gap," IEEE Transactions on Industry Applications, vol. 47 , no. 6, pp. 2495-2504, 2011.

[7] O. Jonah and S. V. Georgakopoulos, "Wireless power transfer in concrete via strongly coupled magnetic resonance," IEEE Transactions on Antennas and Propagation, vol. 61, no. 3, pp. 1378-1384, 2013. 
[8] X. Zhang, Q. Yang, H. Chen, Y. Li, X. Zhang, and L. Jin, "Research on characteristics of frequency splitting in electromagnetic coupling resonant power transmission systems," Proceedings of the Chinese Society of Electrical Engineering, vol. 32, no. 9, pp. 167-172, 2012.

[9] Y. Li, Q. X. Yang, Z. Yan, C. Zhang, and H. Y. Chen, "Analysis on effective range of wireless power transfer and its impact factors," Transactions of China Electrotechnical Society, vol. 28, no. 1, pp. 106-112, 2013.

[10] C. Y. Xia, Y. Sun, N. Jia, Y. Zhuang, and Y. Zhai, "Magnetic circuit parameter optimization for coupled magnetic resonances power transfer system," Transactions of China Electrotechnical Society, vol. 27, no. 11, pp. 139-145, 2012.

[11] L. H. Chen, S. Liu, Y. C. Zhou, and T. J. Cui, "An optimizable circuit structure for high-efficiency wireless power transfer," IEEE Transactions on Industrial Electronics, vol. 60, no. 1, pp. 339-349, 2013.

[12] D. Ahn and S. Hong, "A transmitter or a receiver consisting of two strongly coupled resonators for enhanced resonant coupling in wireless power transfer," IEEE Transactions on Industrial Electronics, vol. 61, no. 3, pp. 1193-1203, 2014.

[13] J. B. Hao, Z. B. Li, and L. P. Zhao, "Genetic algorithm with constraint model for sequencing workingsteps on machining center," Journal of Xian Jiaotong University, vol. 42, no. 7, pp. 860-864, 2008.

[14] C. H. Piao, C. Jiang, H. T. Qiao, C. Cho, and S. Lu, "Modeling and implementation of fixed switching frequency sliding mode control to two-stage DC-DC converter," International Journal of Control, Automation and Systems, vol. 12, no. 6, pp. 1225-1233, 2014.

[15] C. H. Piao, C. Jiang, H. T. Qiao, C. D. Cho, and S. Lu, "Study on the control algorithm of two-stage DC-DC converter for electric vehicles," Advances in Mechanical Engineering, vol. 6, Article ID 203793, 18 pages, 2014.

[16] X. K. Zhu, Study on the equivalent circuit of wireless power transfer system based on Witricity technology [M.S. thesis], Nanchang University, 2012.

[17] K. Deb, "An efficient constraint handling method for genetic algorithms," Computer Methods in Applied Mechanics and Engineering, vol. 186, no. 2-4, pp. 311-338, 2000.

[18] S. H. Tang and Q. J. Zhu, "Effects of the initial population, crossover and mutation rate to the results of genetic algorithms and a possible solution scheme," Bulletin of Science and Technology, vol. 17, no. 3, 2001.

[19] Z. Michalewicz, C. Z. Janikow, and J. B. Krawczyk, "A modified genetic algorithm for optimal control problems," Computers \& Mathematics with Applications, vol. 23, no. 12, pp. 83-94, 1992.

[20] K. Jung, Y. Kim, S. Lim, B. Ahn, and G. Lee, "Effect of friction on critical damage value in compressive deformation," International Journal of Future Engineering, vol. 2015, Article ID 3315112, 5 pages, 2015. 


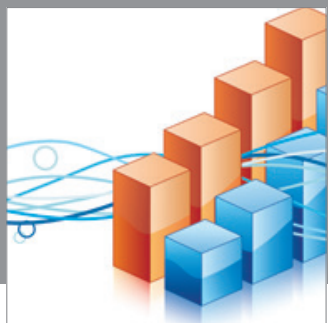

Advances in

Operations Research

mansans

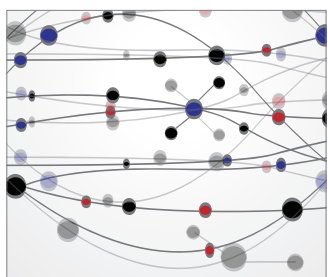

The Scientific World Journal
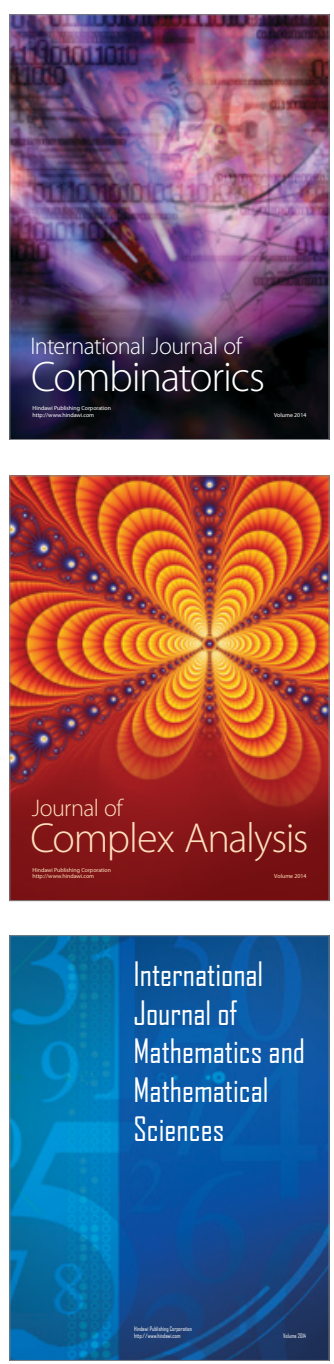
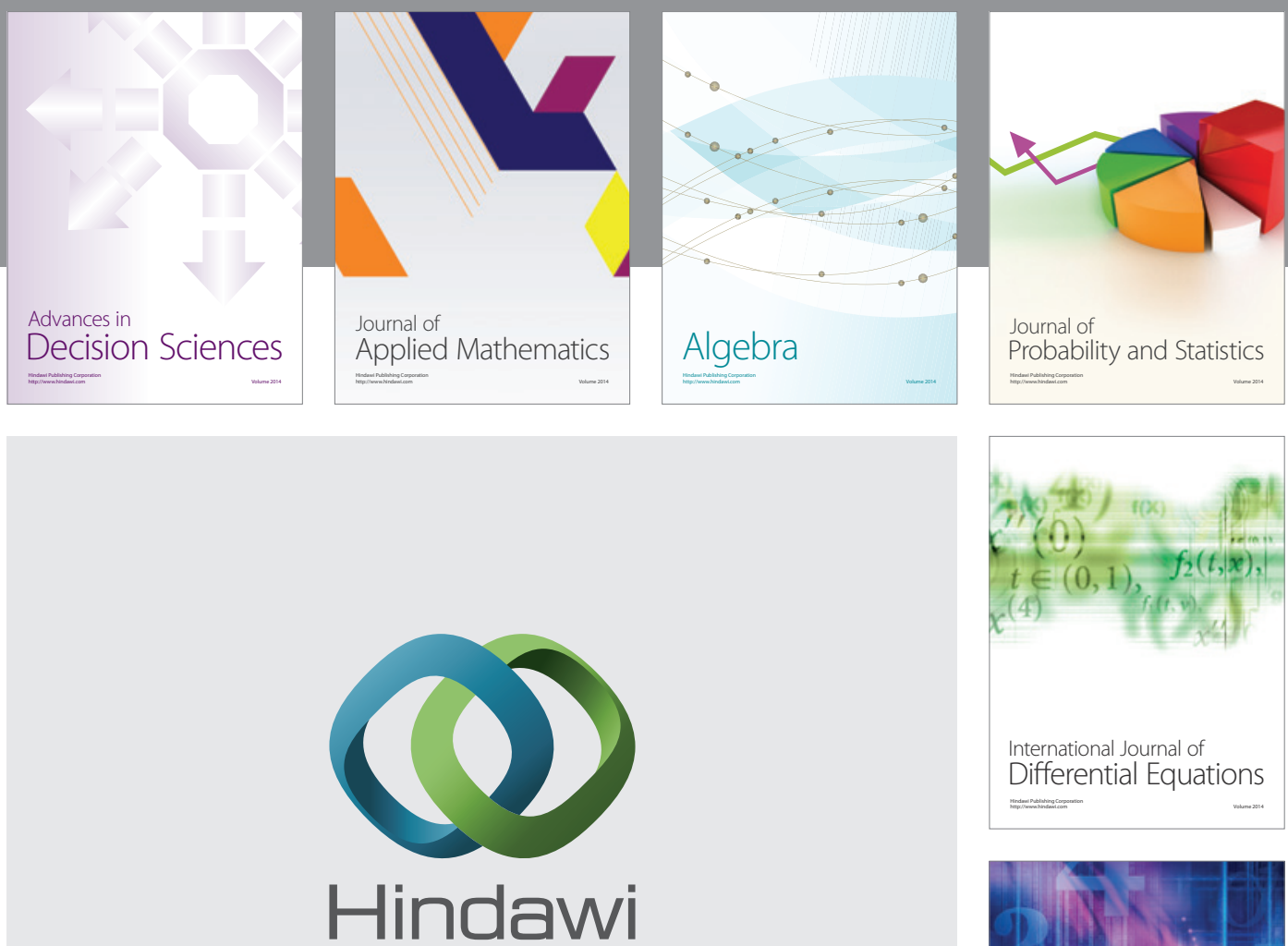

Submit your manuscripts at http://www.hindawi.com
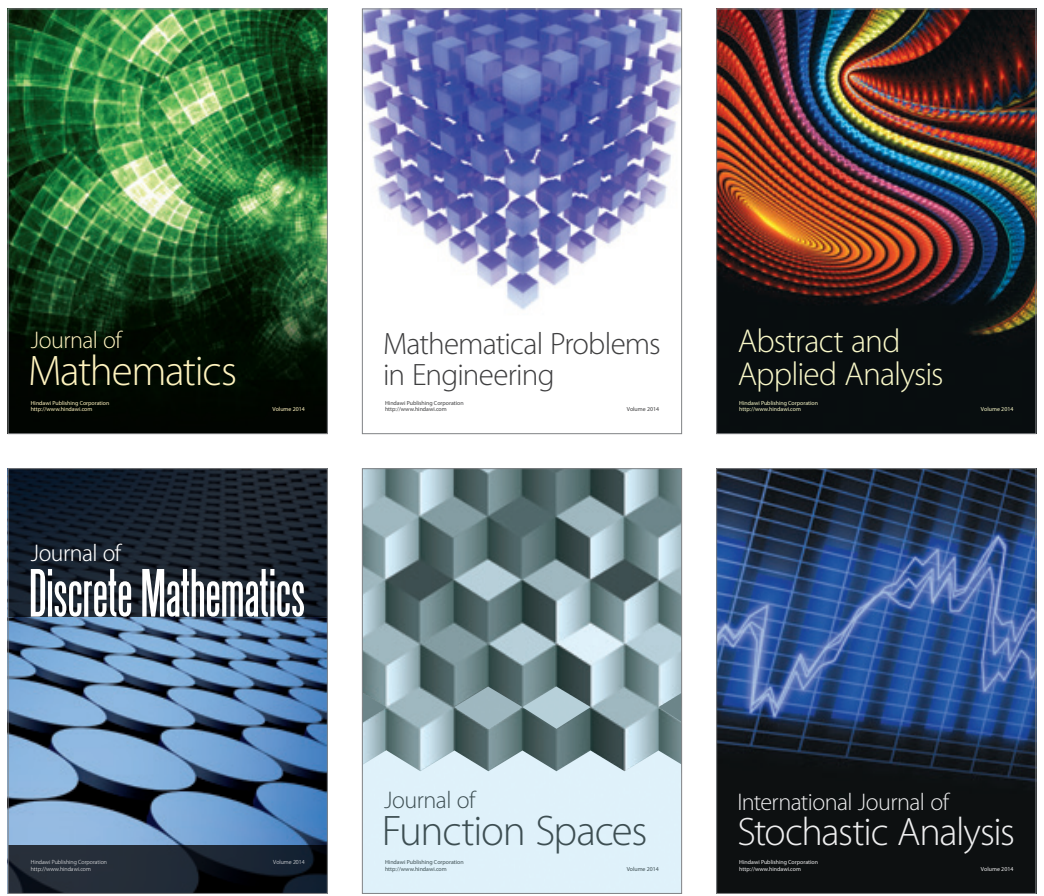

Journal of

Function Spaces

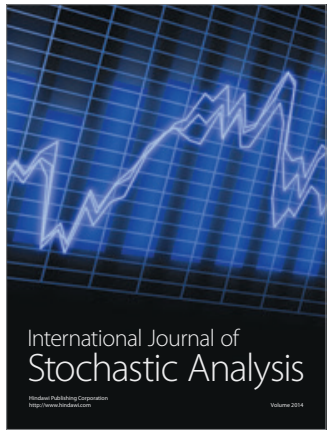

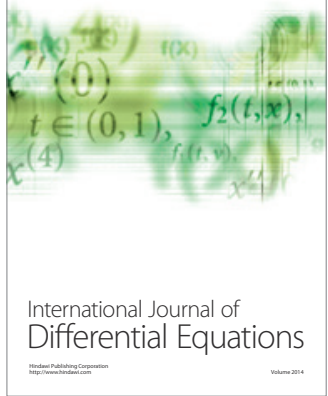
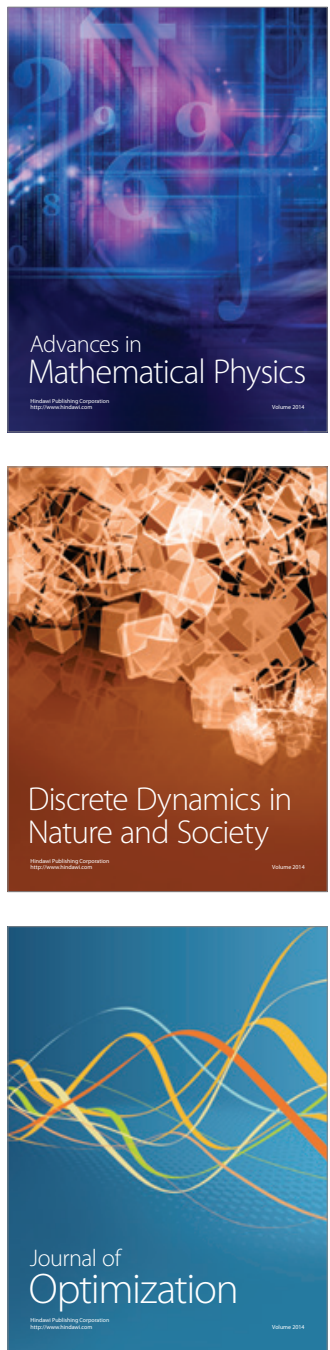